\title{
Stereological Estimate of the Total Number of Neurons in Spinal Segment D9 of the Red-Eared Turtle
}

\author{
Solveig Walløe, ${ }^{1}$ Ulla Vig Nissen, ${ }^{2}$ Rune W. Berg, ${ }^{3}$ Jørn Hounsgaard, ${ }^{3}$ and Bente Pakkenberg ${ }^{1}$ \\ ${ }^{1}$ Research Laboratory for Stereology and Neuroscience, Bispebjerg Hospital, DK-2400 Copenhagen, Denmark, ${ }^{2}$ Clinic for Spinal Cord Injuries, the \\ NeuroScience Centre, Rigshospitalet, University Hospital, DK-2400 Copenhagen, Denmark, and ${ }^{3}$ Department of Neuroscience and Pharmacology, Faculty \\ of Health Sciences, University of Copenhagen, DK-2200 Copenhagen, Denmark
}

The red-eared turtle is an important animal model for investigating the neural activity in the spinal circuit that generates motor behavior. However, basic anatomical features, including the number of neurons in the spinal segments involved, are unknown. In the present study, we estimate the total number of neurons in segment D9 of the spinal cord in the red-eared turtle (Trachemys scripta elegans) using stereological cell counting methods. In transverse spinal cord sections stained with modified Giemsa, motoneurons (MNs), interneurons (INs), and non-neuronal cells were distinguished according to location and morphology. Each cell type was then counted separately using an optical disector with the cell nucleus as counting item. The number of cells in segment D9 was as follows (mean \pm SE): MNs, 2049 \pm 74 ; INs, 16,135 \pm 316 ; non-neuronal cells, 47,504 $\pm 478(n=6)$. These results provide the first estimate of the total number of neurons in a spinal segment in a terrestrial vertebrate based on unbiased stereological methods and an upper bound on the number of neurons involved in segmental sensorimotor activity. These findings also form a crucial quantitative foundation for integrating electrophysiological data into mathematical circuit models.

\section{Introduction}

Animal behavior is orchestrated by functional neural networks in the CNS. The network of neurons that generates a particular behavior can often be accurately delineated and studied in detail in small nervous systems, as exemplified by the network for rhythms in decapod crustaceans (Marder and Bucher, 2007) and the network for crawling and swimming in the leech (Briggman and Kristan, 2006). In contrast, most vertebrate behaviors are generated by large-scale networks of millions of neurons often distributed in several CNS regions. These networks are ill-defined and their overall functional mechanisms hard to study. In this respect, scratching in limbed vertebrates is an exception because the entire sensorimotor transduction is executed by a network of neurons confined to a few spinal segments without the need of other parts of the CNS (Gelfand et al., 1988; Stein, 2005). Scratching has been studied in detail in the cat (Orlovsky et al., 1999) and the turtle in vivo (Robertson and Stein, 1988; Currie and Gonsalves, 1999; Berkowitz, 2001, 2002, 2005; Stein, 2005; Samara and Currie, 2008) but crucially, the spinal cord of the adult turtle is also uniquely amenable to experimentation in vitro (Keifer and Stein, 1983; Hounsgaard and Nicholson, 1990; Currie and Lee, 1996; Alaburda and Hounsgaard, 2003). In vitro experiments provide data of sufficient detail to make mathematical analysis of

\footnotetext{
Received July 28, 2010; revised Dec. 6, 2010; accepted Dec. 10, 2010.

This work was supported by Danish Medical Research Council, The Novo Nordisk Foundation, Lundbeck Foundation, and the Bevica Foundation. We thank Susanne Sørensen for help with the histological methods.

Correspondence should be addressed to Solveig Walløe, Research Laboratory for Stereology and Neuroscience, Bispebjerg University Hospital, Bispebjerg Bakke 23, DK-2400 Copenhagen NV, Denmark. E-mail: swhansen@bio.ku.dk.

DOI:10.1523/JNEUROSCI.3938-10.2011

Copyright $\odot 2011$ the authors $\quad$ 0270-6474/11/312431-05\$15.00/0
}

cell properties and synaptic network activity meaningful (Booth et al., 1997; Svirskis et al., 2000, 2001; Svirskis and Hounsgaard, 2003; Berg et al., 2008). However, quantitative understanding of how minimal mechanosensory stimulation gives rise to complex goal-directed behavior does not emerge from the properties and activity of individual cells in the network alone. Data on network structure is also needed, including specification of cell classes and connectivity. Early anatomical work in the turtle included morphology and organization of hindlimb motoneurons (Ruigrok and Crowe, 1984; Ruigrok et al., 1984, 1985). Insight in the anatomy of the scratch network was provided by studies of the propriospinal projection patterns within the lumbar enlargement (Berkowitz, 2004) and from rostral segments serving the scratch receptive fields (Currie and Stein, 1990; Berkowitz and Stein, 1994). A recent study initiated attempts to quantify the intersegmental projections in the hindlimb enlargement (Nissen et al., 2008). The number of neurons in D9 with intersegmental projections was estimated to be five times higher than the number of motoneurons in the segment. However, the study did not reveal the number of interneurons in D9 with intrasegmental projections only. This is an important number because it provides an upper bound on the number of neurons in the scratch network and a first indication of the relative weight of segmental and intersegmental connections in the lumbar enlargement. For these reasons, we now use stereological quantification to estimate the number of neurons in segment D9 of the lumbar enlargement in the spinal cord of the adult turtle. For comparison we also estimate the number of motoneurons.

\section{Materials and Methods}

Animals. Adult red-eared turtles (Trachemys scripta elegans, $n=6$; gender not determined) of $12-16 \mathrm{~cm}$ carapace length and a weight of $250-$ 


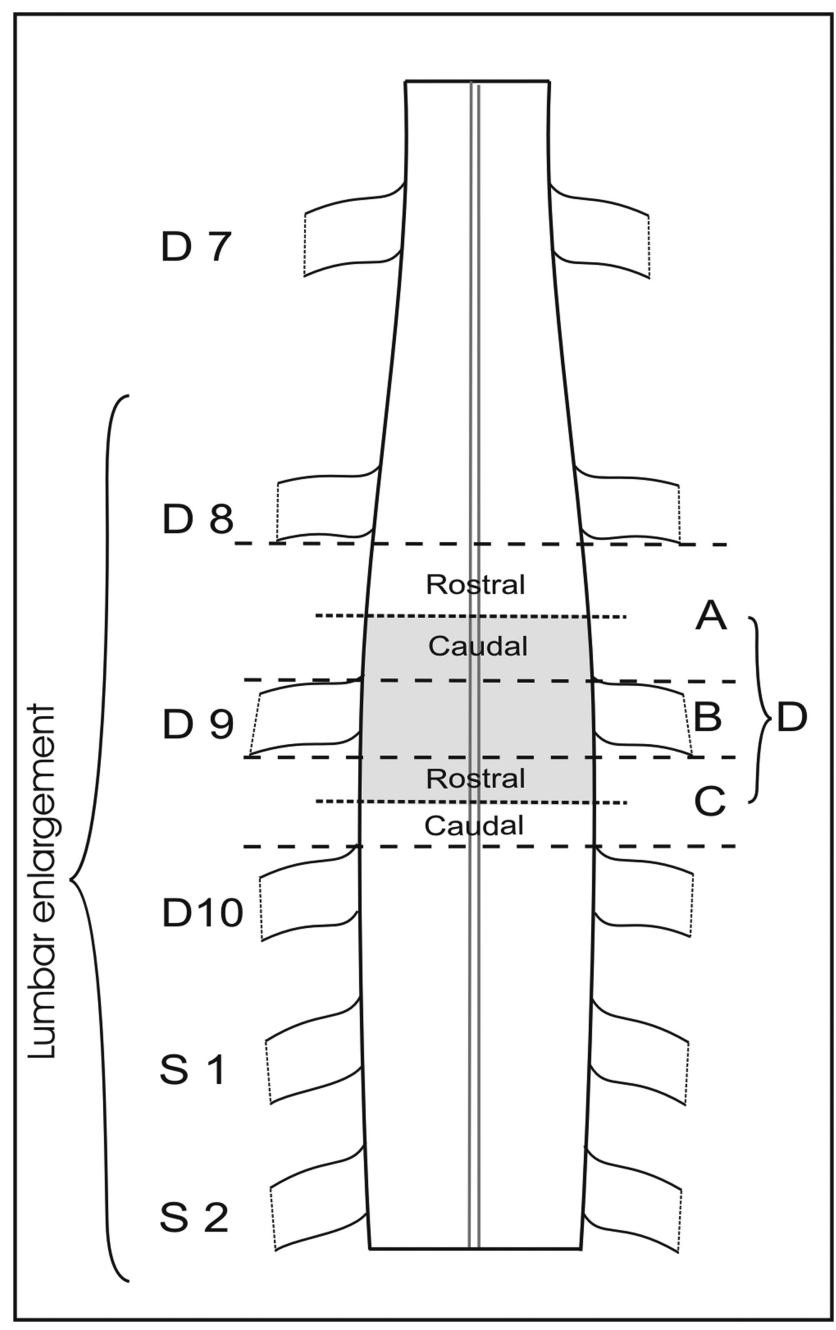

Figure 1. Schematic drawing of the D7 to S2 segments of a turtle spinal cord. Schematic drawing showing the lower turtle spinal cord including the lumbar enlargement; segment D8 to S2. The spinal cord was sectioned from root D8 throughout root D10 and sections identified as belonging to either an interval between roots or within roots. Sections from interval $A$ (between root D8 and D9) were divided into two groups, a rostral group belonging to segment D8 and a caudal group belonging to segment $D 9$. This procedure was also applied for the sections in interval C (between roots D9 and D10). All sections belonging to the D8 or D10 roots as well as those belonging to the rostral D8 and caudal D10 part were left out. Counting was done in sections from interval D (light gray), including sections with roots belonging to D9 (interval B), the caudal part of interval $A$, and rostral part of interval $C$.

$300 \mathrm{~g}$ were anesthetized by intravenous injection of propofol $(30 \mu \mathrm{g} / \mathrm{kg})$ and killed by decapitation (Smith and Perrier, 2006). All blood was removed from the body by perfusion of Ringer solution through the heart [Ringer solution contained the following (in mM): $20 \mathrm{NaCl}, 5 \mathrm{KCl}, 15$ $\mathrm{NaHCO}_{3}, 20 \mathrm{MgCl}_{2}, 3 \mathrm{CaCl}_{2}$, and 20 glucose; saturated with $98 \% \mathrm{O}_{2}$ and $2 \% \mathrm{CO}_{2}$ to obtain $\mathrm{pH}$ 7.6]. The procedure complied with Danish and European legislation on the use of research animals and was approved by the controlling body under the Danish Ministry of Justice. After decapitation, the lumbar part of the spinal cord from segment D6 or D7 to S1 was dissected out of the vertebral column, stripped of pia and dura mater, immersion-fixed in 4\% paraformaldehyde in $0.1 \mathrm{~m} \mathrm{PBS} \mathrm{(pH} \mathrm{7.4),} \mathrm{and}$ stored in the fixative at room temperature for at least $24 \mathrm{~h}$.

Sectioning and staining. Spinal segments D8-D10 were isolated and placed in paraffin. Sections were made on a microtome (Leica Model SM 2400 ) at a setting of $40 \mu \mathrm{m}$ and every second section was mounted on CromAlun-coated glass slides and stained with a modified Giemsa stain (Braendgaard et al., 1990). The sections were divided into those belonging to the interval between roots and those with an identifiable D9 root (Fig. 1). All sections with roots belonging to D9 were used for counting.
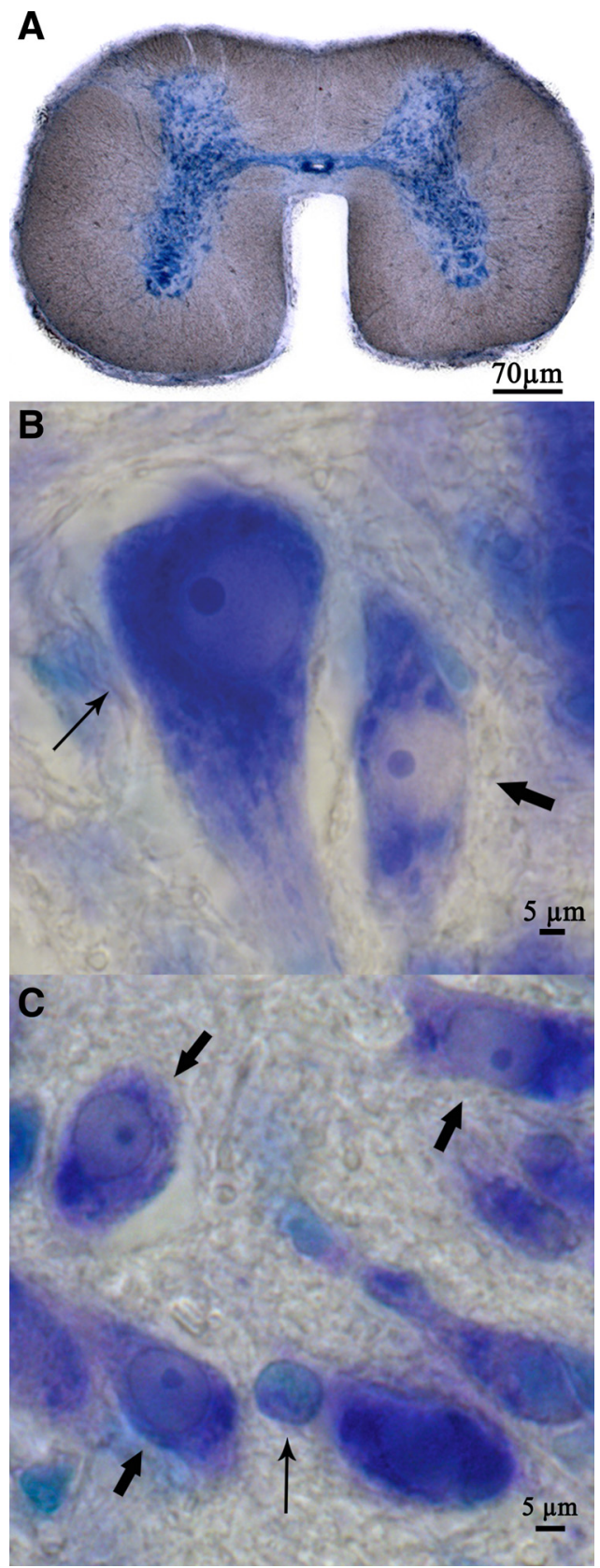

Figure 2. Spinal neurons in the red-eared turtle. $\boldsymbol{A}$, Microphotograph of Giemsa-stained transverse section through spinal segment D9 (ventral side down). $\boldsymbol{B}$, Giemsa image of a motoneuron (thin arrow) and an interneuron (thick arrow). $\boldsymbol{C}$, Giemsa image showing interneurons (thick arrows) and an example of a non-neuronal cell (thin arrow).

In addition, all sections from the interval between root D8 and D9 were divided into two groups, a rostral group belonging to D8 and a caudal group belonging to D9. The same procedure was applied for the sections in the interval between roots D9 and D10. Thus, the total number of sections in the D9 sample consisted of the caudal half of the sections between roots D8 and D9, all sections with D9 rootlets and the rostral half of the sections in the interval between roots D9 and D10 (Fig. 1). In this D9 sample, neurons and non-neuronal cells were counted in every fourth section.

Stereological design and counting procedure. Stereology is an interdisciplinary field that is largely concerned with the three-dimensional interpretation of planar sections of materials or tissues. It provides practical techniques for extracting quantitative information about a threedimensional material from measurements made on two-dimensional 


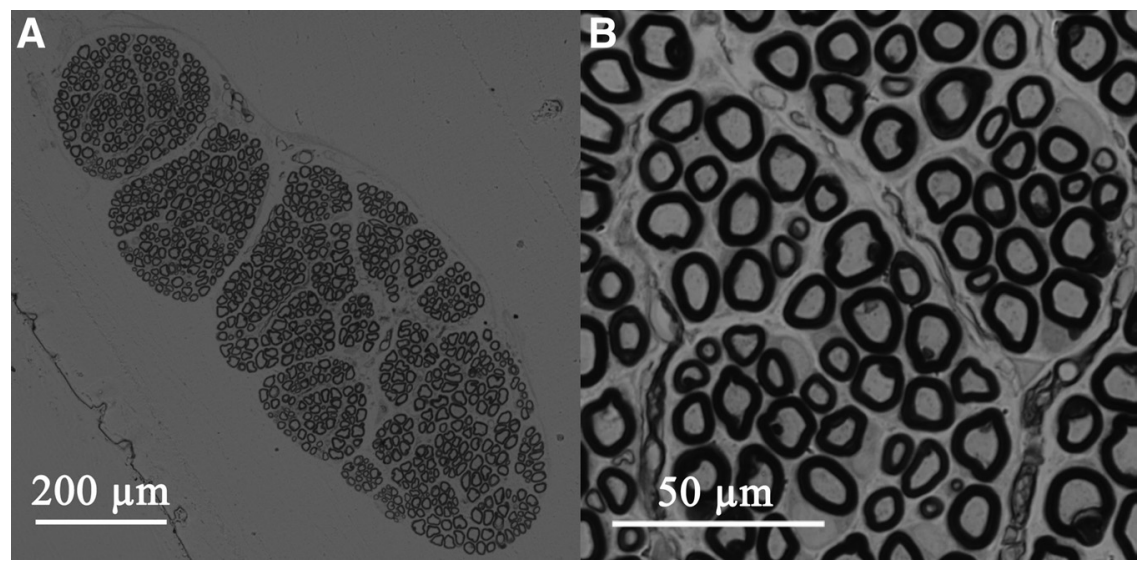

Figure 3. Transverse sections of ventral roots of spinal segment $D 9$. $A, A$ representative overview of a $D 9$ ventral root. $B, A$ corresponding high-power micrograph of a D9 ventral root.

Table 1. Motoneurons, interneurons, and non-neuronal cells in spinal segment D9 of the red-eared turtle

\begin{tabular}{lllll}
\hline & Motoneurons & Interneurons & Non-neuronal cells & IN/MN ratio \\
\hline Case 1 & 1600 & 13,925 & 48,379 & 8.7 \\
Case 2 & 1630 & 15,266 & 49,122 & 9.4 \\
Case 3 & 2016 & 15,580 & 48,959 & 7.7 \\
Case 4 & 2149 & 15,789 & 46,917 & 7.4 \\
Case 5 & 2823 & 16,707 & 41,979 & 5.9 \\
Case 6 & 2079 & 19,541 & 49,668 & 9.4 \\
Mean & 2049 & 16,135 & 47,504 & 7.9 \\
CV & 0.21 & 0.11 & 0.06 & \\
CE & 0.09 & 0.04 & 0.03 &
\end{tabular}

CV, Coefficient of variation (SD/mean); CE, coefficient of error (see text for calculation).

planar sections of the material. Stereology uses random, systematic sampling to provide potentially unbiased and quantitative data and is an important and efficient tool in many applications of microscopy. It may thus provide estimates of cell numbers with a precision determined by the investigators. Stereological estimates are quantitative in nature and the results are unbiased, provided a number of strict requirements to the study design are met (Gundersen and Jensen, 1987; Gundersen et al., 1988a; Howard and Reed, 2005).

A combination of the Cavalieri principle (Gundersen and Jensen, 1987) and the optical disector (Gundersen, 1977) was applied to the actual counting. The equipment used for the optical disector included a microscope (Olympus BX50) with a motorized $x-y$ stage, an electronic microcator (Heidenhain), which was used for measuring movements in the $z$ direction (with precision of $0.5 \mu \mathrm{m}$ ), and the PC software CASTGRID (Visiopharm) for cell counting. The optical disector provided inclusion and exclusion lines to prevent edge effects arising from subsampling (Gundersen, 1977). All cells that came into focus within the disector height were counted, provided they did not touch the exclusion line. The sections were counted with a $60 \times$ oil-immersion objective (final magnification, $2000 \times$ ).

Counting criteria. In this study, the number of motoneurons (MNs), interneurons (INs), and non-neuronal cells in spinal segment D9 were estimated. The cell nucleus was used as the counting item for both neuron types as well as for the non-neuronal cells. Figure $2 \mathrm{~A}$ shows a section through segment D9. Motoneurons were provisionally identified from their morphology, large size, distinct slender and long shape, and location in the ventral half of the ventral horn (Fig. $2 B$ ) - a distinction also used by Nissen et al. (2008). The validity of the motoneurons count was verified by the counts of myelinated axons in D9 ventral roots. INs, scattered in the remaining gray matter throughout the transverse section of the spinal cord, were identified by the presence of a clearly defined nucleus surrounded by a pale cytoplasm and with a dark centrally located nucleolus (Fig. $2 B, C$ ). Non-neuronal cells were identified by their smaller size and their lack of cytoplasm (Fig. 2C). Nonneuronal cells were scattered throughout the region of interest.

To affirm that we could identify the MN and interneurons from other cell types, we applied a specific stain for neurons, NeuN (monoclonal mouse anti-neuronal nuclei antibody; working dilution 1:18,000; Millipore) in one preparation. We estimated the mean density of the interneurons identified by our morphological criterion in Giemsa-stained sections and of immunopositive cells in the NeuN-stained sections and compared. The mean density of neurons was $36.1 \times 10^{6} / \mathrm{cm}^{3}$ in the Giemsasections and $35.3 \times 10^{6} / \mathrm{cm}^{3}$ in NeuN-sections when corrected for shrinkage in the $z$-axis. With the provisional identification of neurons confirmed by NeuN stains, all other cells were classified as non-neuronal cells by exclusion. We have not attempted to identify the nature of the non-neuronal cells.

Total number estimation. The total number of cells in the region of interest was found by multiplying the reference volume, $V_{\text {ref }}$, and the cell density, $N_{\mathrm{V}}$. The cell density is the total number of cells counted in all disectors in a region divided by the total volume in which they are counted (Gundersen et al., 1988b).

Statistical analysis. The precision by which the number of cells was estimated is determined by the coefficient of error, CE. In short, CE is a function of the noise effect and the SURS (systematic uniform random sampling) variance (Gundersen et al., 1999) and it provides information about whether the sampling is sufficiently precise at various levels of the sampling scheme (e.g., sampling of sections, sampling of areas). It is calculated using the following formula:

$$
\mathrm{CE}=\frac{\sqrt{\text { Noise }+\mathrm{VAR}_{\mathrm{SURS}}}}{\sum Q^{-}} .
$$

The Noise effect is the uncertainty in the estimate that comes from disector counts within a section and is equal to $\Sigma Q^{-}$and VAR $\mathrm{VURS}_{\text {SUS }}$ is the uncertainty of sampling between sections, i.e., because repeated estimates based on different set of sections may vary (Gundersen et al., 1999). The sampling is considered optimal when the observed variance of the individual estimate, $\mathrm{CE}^{2}$, is less than half the observed interindividual variance, $\mathrm{CV}^{2}$.

Root counts. Ventral roots D9 were excised from three turtles. The roots were immersion-fixed for $24 \mathrm{~h}$ in $10 \%$ buffet formaldehyde. Specimens were rinsed in cacodylate-buffered $(0.2 \mathrm{M})$, postfixed in $2 \%$ osmium tetroxide in $0.1 \mathrm{~m}$ cacodylate, dehydrated in graded alcohol $(30-100 \%)$, cleared in propylene oxide, and embedded in increasing concentrations of epoxy resin until polymerized in pure epon in a heated cabinet. Cross sections $(1 \mu \mathrm{m})$ were cut with diamond knives, stained with $p$-phenylenediamine, mounted, and photographed. Counts were performed without any sampling, e.g., all myelinated fibers were counted on the photographs (Fig. 3).

\section{Results}

\section{Number of MNs, INs, and non-neuronal cells}

Three types of cells, MNs (Fig. $2 B$ ), INs (Fig. $2 B, C$ ) and nonneuronal cells (Fig. 2C), were counted separately. Table 1 summarizes the mean number of estimated MNs, INs, and non-neuronal cell counts from segment D9.

\section{Number of MNs from root counts}

The mean bilateral number of myelinated fibers in three ventral roots of segment D9 was 2114, which is close to the stereological estimated average number of MN of 2049 (SE, \pm 74 ), $p=0.82$ (unpaired two-tailed Student's $t$ test). 


\section{Discussion}

Functional network activity depends not only on the properties of the neuronal elements and the synapses but also on connectivity and network size (Berg and Hounsgaard, 2009). Using the scratch circuit in the spinal cord of the adult turtle as a model network, the present study provides insight into both these aspects. Segment D9 in the turtle is an integral part of the lumbar network for scratch and swim movements (Berkowitz, 2008). The stereological counting procedure was verified by comparing the estimated average number of MN (2049) in segment D9 to the number of myelinated fibers in the segmental ventral roots (2114). The discrepancy in total number of motoneurons in a previous study (Nissen et al., 2008) may be due in part to the difference in definition of the boarders of segment D9 and in cell counting methods, since the former study was not based on purely unbiased counting methods. Based on the number of myelinated fibers in the ventral roots, each segment in D8-D10 contains 2000-3000 motoneurons (Nissen et al., 2008). Assuming a similar number of segmental motoneurons in segments S1 and S2, we estimate the total number of hindlimb motoneurons in the lumbar enlargement of the turtle to be at least 10,000 . This is much lower than the estimated 60,000 lumbar motoneurons in humans (Tomlinson and Irving, 1977), but surprisingly similar to the number of hindlimb motoneurons in chicks (Hamburger, 1975; Laing, 1982; Tanaka and Landmesser, 1986). In contrast, the number of lumbar motoneurons is $<2000$ in adult bull frogs (Farel et al., 1992) and only 3500 in rodents (Oppenheim, 1986).

Our finding that interneurons in segment D9 outnumber motoneurons by a factor of eight is surprisingly close to the ratio of 5:1 between projecting interneurons and motoneurons in the same segment (Nissen et al., 2008). The estimated number of projecting interneurons is conservative because fluorescent dextran may be a less effective tracer when crystals are applied at the cut surface of the spinal cord rather than the ventral root (Nissen et al., 2008). Thus, the number of projecting interneurons may well be higher than estimated. For this reason, it is possible that only a small fraction of interneurons in D9 have confined intrasegmental projections. These findings demonstrate a physical substrate for extensive short- and long-range connectivity within the scratch network. However, an evaluation of the relative densities of long- and short-range synaptic connections awaits application of techniques recently used in cortical columns (Stepanyants et al., 2009). The ratio of motoneurons to interneurons may be taken as a crude measure of the sensorimotor capabilities of the spinal cord. In this sense, we notice that the ratio for the turtle is close to the ratio of 1:6 extracted from published data aboutthe chick (McKay and Oppenheim, 1991), but lower than the ratio of 1:13-15 extracted from published data about the mouse (Grieshammer et al., 1998; Oppenheim et al., 2000).

To compare the scratch network in the turtle with other functional networks, it is of interest to estimate the number of neurons participating in scratching. The minimal network for rostral and pocket scratch in the adult turtle is contained in segments D8-D10 (Mortin and Stein, 1989). It can be argued that these segments play a similar role in hindlimb scratch as segments L4-L5 in the cat (Mortin and Stein, 1989). Based on the present data and data from Nissen et al. (2008), we estimate that these segments include $\sim 50,000$ neurons. Although scratching in the turtle includes a bilateral core of shared neurons (Stein et al., 1995), it is predominantly a unilateral network activity; i.e., it seems reasonable to assume that an estimated 25,000 ipsilateral neurons are available in hemisegments D8-D10. This undoubt- edly overestimates the number of neurons contributing to the scratch network. An unknown number of neurons in these segments is part of the autonomic nervous system and secondary somatosensory neurons not activated during fictive scratch. All in all, we estimate that the number of neurons in the scratch network is of the same order as the number of neurons in a mammalian cortical column, i.e., $10^{4}$ to $2 \times 10^{4}$ neurons as estimated for a whisker barrel in the rat (Keller and Carlson, 1999; Lübke and Feldmeyer, 2007).

During functional network activity in the cerebral cortex in unanesthetized behaving mammals, neurons enter a high conductance state due to intense concurrent excitatory and inhibitory synaptic activity (Destexhe et al., 2003). Surprisingly, this is also the case in motoneurons and certain spinal interneurons during scratching (Alaburda et al., 2005; Berg et al., 2007). It is tempting to speculate that high conductance states, due to concurrent inhibition and excitation, are structurally inbuilt features of the network in which they occur (Berg and Hounsgaard, 2009). Indeed, this seems to be the case in the cerebral cortex (Kapfer et al., 2007; Silberberg and Markram, 2007). Although similar structural features are unexplored in the spinal cord, we are beginning to delineate the structure of the scratch network in the turtle. Based on the present work, it seems clear that long-range connections are common. A majority of interneurons in D9 project at least to neighboring segments. It is not yet known how far these projections reach, how they organize the functional network modules (Stein, 2005), or the relative proportion of inhibitory and excitatory connections in terms of numbers and synaptic strengths. These crucial questions are subjects of ongoing research.

\section{References}

Alaburda A, Hounsgaard J (2003) Metabotropic modulation of motoneurons by scratch-like spinal network activity. J Neurosci 24:8625-8629.

Alaburda A, Russo R, MacAulay N, Hounsgaard J (2005) Periodic highconductance states in spinal neurons during scratch-like network activity in adult turtles. J Neurosci 25:6316-6321.

Berg RW, Hounsgaard J (2009) Signaling in large-scale neural networks. Cogn Process 10:S9-S15.

Berg RW, Alaburda A, Hounsgaard J (2007) Balanced inhibition and excitation drive spike activity in spinal half-centers. Science 315:390-393.

Berg RW, Ditlevsen S, Hounsgaard J (2008) Intense synaptic activity enhances temporal resolution in spinal motoneurons. PLoS One 3:e3218.

Berkowitz A (2001) Broadly tuned spinal neurons for each form of fictive scratching in spinal turtles. J Neurophysiol 86:1017-1025.

Berkowitz A (2002) Both shared and specialized spinal circuitry for scratching and swimming in turtles. J Comp Physiol A Neuroethol Sens Neural Physiol 188:225-234.

Berkowitz A (2004) Propriospinal projections to the ventral horn of the rostral and caudal hindlimb enlargement in turtles. Brain Res 1014:164-176.

Berkowitz A (2005) Physiology and morphology indicate that individual spinal interneurons contribute to diverse limb movements. J Neurophysiol 94:4455-4470.

Berkowitz A (2008) Physiology and morphology of shared and specialized spinal interneurons for locomotion and scratching. J Neurophysiol 99:2887-2901.

Berkowitz A, Stein PS (1994) Activity of descending propriospinal axons in the turtle hindlimb enlargement during two forms of fictive scratching: broad tuning to regions of the body surface. J Neurosci 14:5089-5104.

Booth V, Rinzel J, Kiehn O (1997) Compartmental model of vertebrate motoneurons for $\mathrm{Ca} 2+$-dependent spiking and plateau potentials under pharmacological treatment. J Neurophysiol 78:3371-3385.

Braendgaard H, Evans SM, Howard CV, Gundersen HJ (1990) The total number of neurons in the human neocortex unbiasedly estimated using optical disectors. J Microsc 157:285-304.

Briggman KL, Kristan WB Jr (2006) Imaging dedicated and multifunctional neural circuits generating distinct behaviors. J Neurosci 26:10925-10933. 
Currie SN, Gonsalves GG (1999) Reciprocal interactions in the turtle hindlimb enlargement contribute to scratch rhythmogenesis. J Neurophysiol 81:2977-2987.

Currie SN, Lee S (1996) Sensory-evoked pocket scratch motor patterns in the in vitro turtle spinal cord: reduction of excitability by an $\mathrm{N}$-methylD-aspartate antagonist. J Neurophysiol 76:81-92.

Currie SN, Stein PS (1990) Cutaneous stimulation evokes long-lasting excitation of spinal interneurons in the turtle. J Neurophysiol 64:1134-1148.

Destexhe A, Rudolph M, Paré D (2003) The high-conductance state of neocortical neurons in vivo. Nat Rev Neurosci 4:739-751.

Farel PB, St Wecker PG, Wray SE (1992) Neuron addition in the postmetamorphic frog. Exp Gerontol 27:111-124.

Gelfand IM, Orlovsky GN, Shik ML (1988) Locomotion and scratching in tetrapods. In: Neural control of rhythmic movements in vertebrates (Cohen AH, Rossignol S, Grillner S, eds), pp 167-199. New York: Wiley.

Grieshammer U, Lewandoski M, Prevette D, Oppenheim RW, Martin GR (1998) Muscle-specific cell ablation conditional upon Cre-mediated DNA recombination in transgenic mice leads to massive spinal and cranial motoneuron loss. Dev Biol 197:234-247.

Gundersen HJ (1977) Notes on the estimation of the numerical density of arbitrary profiles: the edge effect. J Microscopy 111:219-222.

Gundersen HJ, Jensen EB (1987) The efficiency of systematic sampling in stereology and its prediction. J Microsc 147:229-263.

Gundersen HJ, Bendtsen TF, Korbo L, Marcussen N, Møller A, Nielsen K, Nyengaard JR, Pakkenberg B, Sørensen FB, Vesterby A (1988a) Some new, simple and efficient stereological methods and their use in pathological research and diagnosis. APMIS 96:379-394.

Gundersen HJ, Bagger P, Bendtsen TF, Evans SM, Korbo L, Marcussen N, Møller A, Nielsen K, Nyengaard JR, Pakkenberg B (1988b) The new stereological tools: disector, fractionator, nucleator and point sampled intercepts and their use in pathological research and diagnosis. APMIS 96:857-881.

Gundersen HJ, Jensen EB, Kiêu K, Nielsen J (1999) The efficiency of systematic sampling in stereology—reconsidered. J Microsc 193:199-211.

Hamburger V (1975) Cell death in the development of the lateral motor column of the chick embryo. J Comp Neurol 160:535-546.

Hounsgaard J, Nicholson C (1990) The isolated turtle brain and the physology of neuronal circuits. In: Preparation of vertebrate central nervous systems in vitro (Jahnsen H, ed), pp 155-181. New York: Wiley.

Howard V, Reed MG (2005) Unbiased stereology: three-dimensional measurement in microscopy. BIOS Scientific.

Kapfer C, Glickfeld LL, Atallah BV, Scanziani M (2007) Supralinear increase of recurrent inhibition during sparse activity in the somatosensory cortex. Nat Neurosci 10:743-753.

Keifer J, Stein PS (1983) In vitro motor program for the rostral scratch reflex generated by the turtle spinal-cord. Brain Res 266:148-151.

Keller A, Carlson GC (1999) Neonatal whisker clipping alters intracortical, but not thalamocortical projections, in rat barrel cortex. J Comp Neurol 412:83-94.

Laing NG (1982) Motor projection patterns to the hind limb of normal and paralysed chick embryos. J Embryol Exp Morphol 72:269-286.

Lübke J, Feldmeyer D (2007) Excitatory signal flow and connectivity in a cortical column: focus on barrel cortex. Brain Struct Funct 212:3-17.

Marder E, Bucher D (2007) Understanding circuit dynamics using the stomatogastric nervous system of lobsters and crabs. Annu Rev Physiol 69:291-316.

McKay SE, Oppenheim RW (1991) Lack of evidence for cell death among avian spinal cord interneurons during normal development and following removal of targets and afferents. J Neurobiol 22:721-733.
Mortin LI, Stein PS (1989) Spinal cord segments containing key elements of the central pattern generators for three forms of scratch reflex in the turtle. J Neurosci 9:2285-2296.

Nissen UV, Moldovan M, Hounsgaard J, Glover JC (2008) Organization of projection-specific interneurons in the spinal cord of the red-eared turtle. Brain Behav Evol 72:179-191.

Oppenheim RW (1986) The absence of significant postnatal motoneuron death in the brachial and lumbar spinal cord of the rat. J Comp Neurol 246:281-286.

Oppenheim RW, Houenou LJ, Parsadanian AS, Prevette D, Snider WD, Shen L (2000) Glial cell line-derived neurotrophic factor and developing mammalian motoneurons: regulation of programmed cell death among motoneuron subtypes. J Neurosci 20:5001-5011.

Orlovsky GN, Deliagina TG, Grillner S (1999) Neuronal control of locomotion: from mollusc to man. Oxford UP.

Robertson GA, Stein PS (1988) Synaptic control of hindlimb motoneurones during three forms of fictive scratch reflex in the turtle. J Physiol 404:101-128.

Ruigrok TJ, Crowe A (1984) The organization of motoneurons in the turtle lumbar spinal cord. J Comp Neurol 228:24-37.

Ruigrok TJ, Crowe A, ten Donkelaar HJ (1984) Morphology of lumbar motoneurons innervating hindlimb muscles in the turtle Pseudemys scripta elegans: an intracellular horseradish peroxidase study. J Comp Neurol 230:413-425.

Ruigrok TJ, Crowe A, Ten Donkelaar HJ (1985) Morphology of primary afferents to the spinal cord of the turtle Pseudemys scripta elegans. Anat Embryol (Berl) 171:75-81.

Samara RF, Currie SN (2008) Location of spinal cord pathways that control hindlimb movement amplitude and interlimb coordination during voluntary swimming in turtles. J Neurophysiol 99:1953-1968.

Silberberg G, Markram H (2007) Disynaptic inhibition between neocortical pyramidal cells mediated by Martinotti cells. Neuron 53:735-746.

Smith M, Perrier JF (2006) Intrinsic properties shape the firing pattern of ventral horn interneurons from the spinal cord of the adult turtle. J Neurophysiol 96:2670-2677.

Stein PS (2005) Neuronal control of turtle hindlimb motor rhythms. J Comp Physiol A Neuroethol Sens Neural Behav Physiol 191:213-229.

Stein PS, Victor JC, Field EC, Currie SN (1995) Bilateral control of hindlimb scratching in the spinal turtle: contralateral spinal circuitry contributes to the normal ipsilateral motor pattern of fictive rostral scratching. J Neurosci 15:4343-4355.

Stepanyants A, Martinez LM, Ferecskóo AS, Kisvárday ZF (2009) The fractions of short- and long-range connections in the visual cortex. Proc Natl Acad Sci U S A 106:3555-3560.

Svirskis G, Hounsgaard J (2003) Influence of membrane properties on spike synchronization in neurons: theory and experiments. Network 14:747-763.

Svirskis G, Hounsgaard J, Gutman A (2000) Detection of inhomogeneities in membrane ohmic resistance in geometrically complex systems. Membr Cell Biol 14:413-420.

Svirskis G, Gutman A, Hounsgaard J (2001) Electrotonic structure of motoneurons in the spinal cord of the turtle: inferences for the mechanisms of bistability. J Neurophysiol 85:391-398.

Tanaka H, Landmesser LT (1986) Cell death of lumbosacral motoneurons in chick, quail, and chick-quail chimera embryos: a test of the quantitative matching hypothesis of neuronal cell death. J Neurosci 6:2889-2899.

Tomlinson BE, Irving D (1977) The numbers of limb motor neurons in the human lumbosacral cord throughout life. J Neurol Sci 34:213-219. 